\title{
Left imaginal neglect in heminattention: experimental study with the O'clock Test
}

\author{
D. Grossi', R. Angelini', A. Pecchinenda² and L. Pizzamiglio ${ }^{2,3}$ \\ 'Department of Neurology, II Medical School, University of Naples, "Federico II", \\ ${ }^{2}$ Department of Psychology, University of Rome, "La Sapienza", and ${ }^{3}$ S. Lucia Clinic, \\ Rome, Italy
}

Correspondence to: D. Grossi, Clinica Neurologica, II Facoltà di Medicina e Chirurgia, Via Sergio Pansini, 5, 80131, Napoli, Italy

\begin{abstract}
Both sensory and imaginal defects have been reported in unilateral neglect, but their assessment based on the description of famous squares can be difficult in a clinical setting. The $O$ 'clock Test is an alternative tool for revealing imaginal defects. Our aim was to demonstrate imaginal neglect in patients with left heminattention. Ten patients were studied and a mild unilateral defect in imaginal processes was found with an increase in the defect when the patients were fatigued.
\end{abstract}

Keywords: Heminattention - Mental imagery

\section{INTRODUCTION}

Impairments due to heminattention are not limited to defects in sensory perceptions, but may also include disturbances in spatial representations, as demonstrated by Bisiach and Luzzatti (1978) and Bisiach et al. (1979).

Recently, Grossi et al. (1989) studied the different roles of the cerebral hemispheres in mental imagery using the O'clock Test, which contains closely comparable perceptual and imaginal tasks. In this test the subject is asked to imagine a pair of clock faces indicating different times, proposed verbally by the examiner; then he is asked to indicate which clock hands define the larger angle. The proposed times have one hand along the vertical midline and the other in the right or left half of the clock face. An analogous test was given to test perception.

One of two patients tested by Grossi et al. had severe heminattention. He demonstrated left neglect in both perceptual and imaginal tasks, supporting the hypothesis that the same spatial representational system is shared by perceptual and imaginal processing. Moreover, the authors suggested that the O'clock Test is more useful than the description of famous squares reported by Bisiach and Luzzatti (1978), since the latter is difficult to administer to patients in the clinical setting. In fact, not all home cities of the patients are well known by the examiners. Furthermore, very often the description is easily influenced by semantic information.

The aim of the present study was to administer the
O'clock Test to a group of patients with visual heminattention in order to examine its sensitivity in detecting imaginal neglect.

\section{METHODS}

\section{Subjects}

Twenty-four patients with heminattention were examined, but only 10 were able to perform the experimental tasks completely. Seven patients were unable to perform correctly the preliminary task described later, three refused to complete the imaginal task because of easy fatiguing, and four could not participate because of co-existing medical problems.

The group of patients who completed the experiment consisted of six males and four females: average age was 67 (S.D. = 11.76); mean years of schooling, 7.6 (S.D. = 4.27); and average disease duration, 49.4 months (S.D. $=99.8)$. All the patients had damage to the posterior right hemisphere.

Heminattention was tested using: (1) Sentence reading (Pizzamiglio et al., 1989); (2) Barrage Test (Albert, 1973); (3) Wundt-Jastrow Test (Massironi et al., 1988); and (4) Letter Cancellation Test (Diller et al., 1980). The patients' scores indicated mild-moderate neglect impairment. 


\section{O'clock Test}

We adopted a version of the original test proposed by Grossi et al. (1989) (see Appendix 1).

Preliminary task. Subjects were requested to turn the hands of a clock to the time indicated by the examiner. There were 16 items indicating times when the hands are in the left half of the clock face (i.e. 9.30), called the left condition (LC), and 16 in the right half (i.e. 3.00) called the right condition (RC). The aim of this task was to determine whether the patient could read an analogical clock.

Perceptual task. This test involved 32 pairs of figures representing clock faces indicating different times, 16 pairs in the RC and 16 pairs in the LC. The figures were arranged in vertical columns to avoid errors due to heminattention. For each pair, the patients were requested to indicate the clock face on which the hands formed the larger angle.

Imaginal task. Patients were requested to imagine a pair of clock faces indicating the different times which were proposed verbally by the examiner (i.e. 9.30 and 10.00). They were then asked to indicate on which of the clocks the hands defined the larger angle. To eliminate memory difficulties, the times were written on a sheet of paper. Twenty RC and 20 LC were administered.

In order to control some variables, the following criteria were adopted in setting up the task:

(1) We selected only times involving half hours (i.e. $7.30,8.30,5.30$ ) or hours (i.e. $7.00,9.00,5.00$ ) to avoid times that were too difficult to imagine, such as 5.25 or 6.27 .

(2) The times chosen for the RC and LC were selected in order to make left and right items equivalent in difficulty.

(3) The pairs of time were matched to compare size angle; namely, there were the same number of different pairs for $30^{\circ}, 60^{\circ}, 90^{\circ}$, and $120^{\circ}$ in both conditions.

(4) In both conditions we also matched pairs in which the correct answers corresponded to numerically greater times (i.e. 3.00 vs 1.00 ) with pairs in which they did not correspond (i.e. 7.00 vs 11.00 ) in order to avoid patients considering only the numerically greater times.

\section{Procedure}

Patients first performed the preliminary task. If this was performed correctly (cut-off of $90 \%$ correct responses), they were administered the other two tasks. In this way, we selected only the patients able to read an analogical clock both in its left and right half. Consequently, some patients $(n=7)$ were excluded from the experiment because the nature of a defect in the imaginal task would have been difficult to interpret.

The imaginal task was given in four blocks, containing
10 pairs each (five pairs of RC and five pairs of LC); the patient rested for several minutes following each block. In order to obtain a large number of comparisons, the imaginal task was administered again, in two separate sessions, with a different block order.

\section{RESULTS}

The patients' scores are summarized in Table I. A $t$-test revealed that preliminary and perceptual tasks were performed without significant differences in either condition (RC and LC; $p=0.1206$ and $p=0.1679$, respectively), while the imaginal task (first session) showed a significant difference $(p=0.0296)$. The other experimental sessions (second and third) confirmed that the imaginal task was performed worse in $\mathrm{RC}(p=0.033$ and $p=0.0375$, respectively).

Figure 1 clearly shows that, while performance on the $\mathrm{RC}$ remains constant across the three sessions, moving from the first to the fourth block, the LC tends to progressively decrease within each session. It is noteworthy that all patients showed this effect. Consequently, the imaginal test data were also elaborated with a three-way analysis of variance for repeated measures: condition (RC or LC) by order of presentation (blocks 1, 2, 3 and 4 across the three sessions) and by session (1-3).

Statistical analysis revealed a significant effect for "condition" ( $\mathrm{F}=9.846 ; p=0.012)$ and "order of presen-

TABLE I. Mean correct scores of the patients

\begin{tabular}{lccc}
\hline & Left condition & Right condition & Max. score \\
\cline { 1 - 4 } Preliminary task & $14.70(1.48)$ & $15.50(0.67)$ & 16 \\
Perceptual task & $15.00(0.89)$ & $15.60(0.66)$ & 16 \\
Imaginal task & & & \\
$\quad$ First session & $12.50(1.51)$ & $14.50(2.32)$ & 20 \\
Second session & $12.40(1.27)$ & $14.00(2.26)$ & 20 \\
Third session & $12.20(2.44)$ & $14.30(2.95)$ & 20 \\
\hline
\end{tabular}

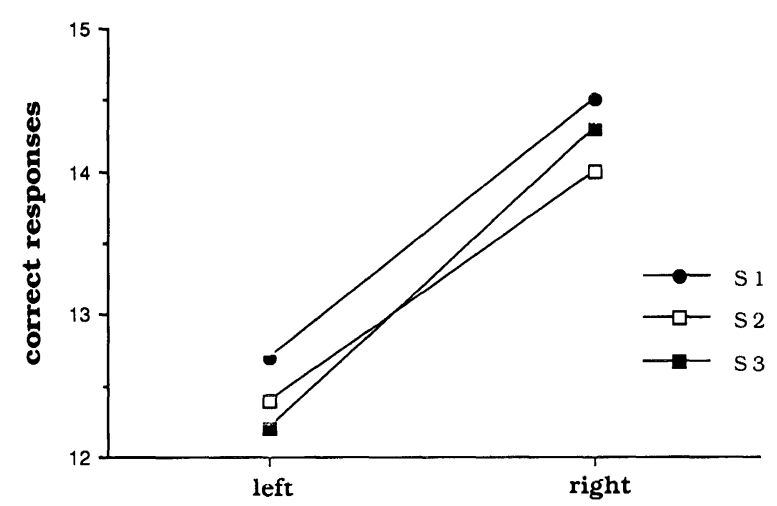

FIG. 1. Mean correct score in imaging the left and the right clock face for the three sessions (S1, S2, S3). 


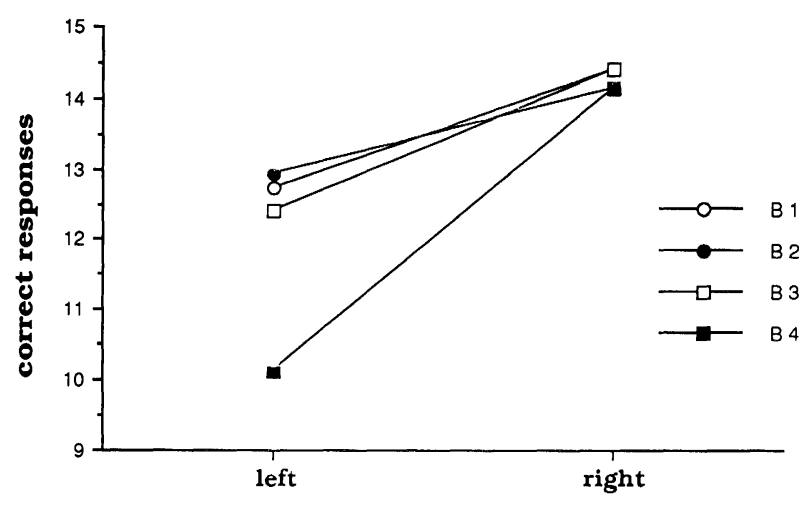

FIG. 2. Mean correct responses for the blocks (B1, B2, B3, B4) to the left and to the right of the imaged clock face.

tation" ( $\mathrm{F}=4.683 ; p=0.0093)$. The interaction "condition $\times$ order of presentation" was also significant $(\mathrm{F}=5.864 ; p=0.0032)$. The variable "session" was not significant.

Post hoc comparison using Duncan's test shows a significant difference between RC and LC for block 3 $(p<0.05)$ and a numerically greater one for block 4 $(p<0.01)$.

\section{DISCUSSION}

In summary, the patients revealed a significant defect in the imaginal processes necessary for mentally matching pairs of times with hands located in the left half of a clock face. The measure of this defect is stable and easily replicated in the same patients in different testing sessions. In this sense, the present test can be considered useful to detect disorders of visual imagery without requiring the patient to produce images from his long-term memory which would be difficult to control for appropriateness (i.e. place descriptions). However, the test presents some constraints. The tasks have a chance level of 0.50 ; this implies that $50 \%$ of correct solutions can be obtained by guessing. Consequently, in individual assessment differences in imaginal task may not be significant because they are too small. This restriction can be partially overcome by repeating the task many times in different sessions.

The imaginal neglect for the left side becomes progressively more evident during the course of the experiment and is most apparent at the presentation of the last block of pairs. This effect cannot be related to the kinds of items because the succession of blocks was systematically varied in every session.

It is also relevant that although affected by heminattention, all patients performed the perceptual task well, in contrast with Grossi et al.'s (1989) patient who was unable to perform the perceptual task in the LC. This result may be explained simply in terms of different degrees of difficulty of the tasks in less impaired patients, or it could imply activation of different functional components. A tendency towards a significant difference also in perceptual tasks seems to be present in our work (Table I).

The present data further support the idea that an imaginal defect for the left side of mental images can be systematically found in a group of neglect patients using an appropriate technique such as the O'clock Test. The constancy of all patients' results in the three sessions demonstrates the effectiveness of the O'clock Test in detecting imaginal neglect. By repeating the testing procedure several times within the same session, i.e. when the patient is more fatigued by repeated efforts, the defect for the left imaginal space can be clearly outlined.

The interpretation of the tiring effect revealed only in the LC is difficult to understand on the basis of the model of mental image (Farah, 1984; Kosslyn, 1987; Grossi et al., 1989). Imaginal neglect is not always detectable in heminattention patients; this could be related to the fact that imaginal processing requires effort (Hasher and Zachs, 1984) and patients' strong control may prevent its clinical evidence; with fatigue control may decrease unmasking the imaginal impairment. Our results seem to support the hypothesis that imaginal defect does not occur as a result of generating mental images (i.e. clock faces) or in matching them, but in functional components underlying these activities (i.e. attentive control and resources) which are mainly impaired in left perceptive and imaginal space.

\section{REFERENCES}

Albert ML (1973) A simple test of visual neglect. Neurology, 23, 658-664.

Bisiach E and Luzzatti C (1978) Unilateral neglect of representational space. Cortex, 14, 129-133.

Bisiach E, Luzzatti C and Perani C (1979) Unilateral neglect, representational schema and consciousness. Brain, 102, 609-618.

Diller L, Weinberg J, Piatesky E, Ruckdeschel-Hibbard M, Egelko S, Scotzin M, Commotakis J and Gordon M (1980) Methods for the Evaluation and Treatment of the Visual Perceptual Difficulties of Right Brain Damaged Individuals. Supplement of the 8th Annual Workshop for Rehabilitation Professionals, New York University Medical Center, New York.

Farah MJ (1984) The neurologic basis of mental imagery: A componential analysis. Cognition, 18, 245-272.

Grossi D, Modafferi A, Pelosi L and Trojano L (1989) On the different roles of the cerebral hemispheres in mental imagery: The "O'clock Test" in two clinical cases. Brain and Cognition, 10, 18-27.

Hasher L and Zachs RT (1984) Automatic processing of fundamental information: The case of frequency of occurrence. American Psychologist, 39, 1372-1388. 
Kosslyn SM (1987) Seeing and imagining in the cerebral hemispheres: A computational approach. Psychological Review, 94, 148-175.

Massironi M, Antonucci G, Pizzamiglio L, Vitale MV and Zoccolotti P (1988) The Wundt-Jastrow illusion in the study of spatial hemi-inattention. Neuropsychologia, 26, 161-166.
Pizzamiglio L, Judica A, Razzano C and Zoccolotti P (1989) Toward a comprehensive diagnosis of visuo-spatial disorders in unilateral brain damaged patients. Psychological Assess-

(Received 14 July 1993; accepted 6 August 1993

APPENDIX 1. O'clock Test

\begin{tabular}{|c|c|c|c|c|c|c|c|}
\hline \multicolumn{8}{|c|}{ Preliminary task } \\
\hline 1 & 3.00 & 9 & 3.00 & 17 & 7.00 & 25 & 8.00 \\
\hline 2 & 10.30 & 10 & 2.30 & 18 & 8.30 & 26 & 3.30 \\
\hline 3 & 1.30 & 11 & 11.30 & 19 & 2.00 & 27 & 7.30 \\
\hline 4 & 7.00 & 12 & 9.00 & 20 & 11.00 & 28 & 2.00 \\
\hline 5 & 11.30 & 13 & 1.30 & 21 & 10.30 & 29 & 5.30 \\
\hline 6 & 5.00 & 14 & 7.00 & 22 & 1.00 & 30 & 10.00 \\
\hline 7 & 2.30 & 15 & 10.30 & 23 & 4.30 & 31 & 9.30 \\
\hline 8 & 9.00 & 16 & 5.00 & 24 & 5.00 & 32 & 4.00 \\
\hline \multicolumn{8}{|c|}{ Perceptual task } \\
\hline 1 & $\underline{11.30 / 7.30}$ & 9 & $1.30 / 1.00$ & 17 & $11.00 / \underline{7.00}$ & 25 & $5.30 / \underline{5} .00$ \\
\hline 2 & $1.30 / 5.30$ & 10 & $11.30 / 11.00$ & 18 & $1.00 / \overline{5.00}$ & 26 & $7.30 / \overline{7.00}$ \\
\hline 3 & $4.30 / \underline{5.00}$ & 11 & $7.30 / 10.30$ & 19 & $1.30 / 4.30$ & 27 & $11.00 / 10.30$ \\
\hline 4 & $10.00 / 10.30$ & 12 & $4.30 / 4.00$ & 20 & $9.00 / 7.30$ & 28 & $\underline{1.30 / 3.00}$ \\
\hline 5 & $7.00 / 8.30$ & 13 & $\underline{2.30 / 5.30}$ & 21 & $8.30 / 11.30$ & 29 & $1.00 / \underline{2.30}$ \\
\hline 6 & $2.00 / 2.30$ & 14 & $8.00 / 8.30$ & 22 & $5.30 / 3.00$ & 30 & $11.30 / 9.00$ \\
\hline 7 & $9.30 / 8.00$ & 15 & $3.30 / 2.00$ & 23 & $10.30 / 9.00$ & 31 & $\underline{2.00 / 5.30}$ \\
\hline 8 & $4.00 / 3.30$ & 16 & $10.00 / \underline{9.30}$ & 24 & $\underline{2.30 / 3.00}$ & 32 & $\underline{10.00 / 7.30}$ \\
\hline \multicolumn{8}{|c|}{ Imaginal task } \\
\hline 1 & $\underline{1.30 / 1.00}$ & 11 & $\underline{7.00 / 7.30}$ & 21 & $\underline{1.30} / 4.30$ & 31 & $10.30 / 11.00$ \\
\hline 2 & $\overline{7.30} / 10.30$ & 12 & $1.30 / 2.00$ & 22 & $1 \overline{1.00 / 7.00}$ & 32 & $1.30 / 5.30$ \\
\hline 3 & $2.00 / 2.30$ & 13 & $11.00 / \underline{9} .30$ & 23 & $1.00 / \underline{5.00}$ & 33 & $\underline{11.30 / 7.30}$ \\
\hline 4 & $11.30 / 11.00$ & 14 & $5.30 / \underline{5.00}$ & 24 & $5.00 / 3.30$ & 34 & $7.30 / 9.00$ \\
\hline 5 & $10.00 / 10.30$ & 15 & $1.00 / \underline{3} .30$ & 25 & $8.30 / 11.30$ & 35 & $1.00 / 2.30$ \\
\hline 6 & $2.30 / 5.30$ & 16 & $10.00 / 11.30$ & 26 & $3.00 / 2.30$ & 36 & $9.00 / 8.30$ \\
\hline 7 & $1.00 / \underline{4.30}$ & 17 & $7.30 / 10.30$ & 27 & $\underline{7.00 / 9.30}$ & 37 & $\overline{3.00} / 5.30$ \\
\hline 8 & $7.30 / 10.00$ & 18 & $2.00 / \underline{3.30}$ & 28 & $9.00 / \underline{10.30}$ & 38 & $11.30 / 8.00$ \\
\hline 9 & $\underline{2.00 / 5.30}$ & 19 & $\underline{9.30 / 10.00}$ & 29 & $4.30 / \underline{3.30}$ & 39 & $4.00 / 1.30$ \\
\hline 10 & $8.30 / 11.00$ & 20 & $5.00 / 2.30$ & 30 & $8.00 / \overline{9} .30$ & 40 & $3.00 / 4.30$ \\
\hline
\end{tabular}

Correct responses are underlined. 


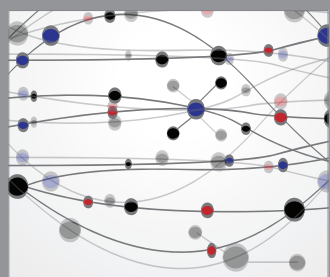

The Scientific World Journal
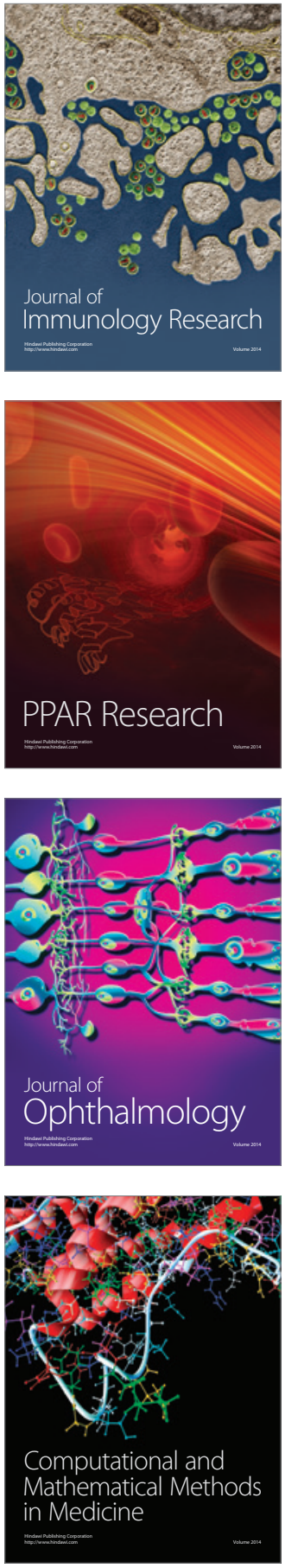

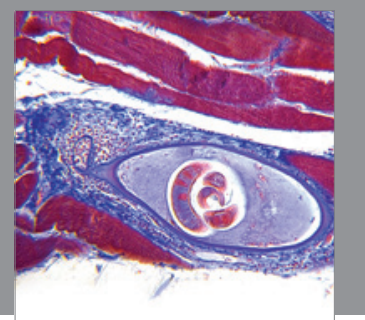

Gastroenterology

Research and Practice
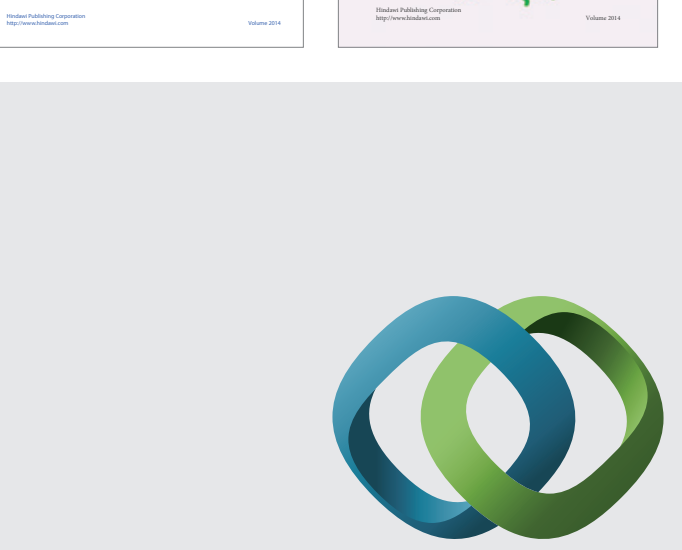

\section{Hindawi}

Submit your manuscripts at

http://www.hindawi.com
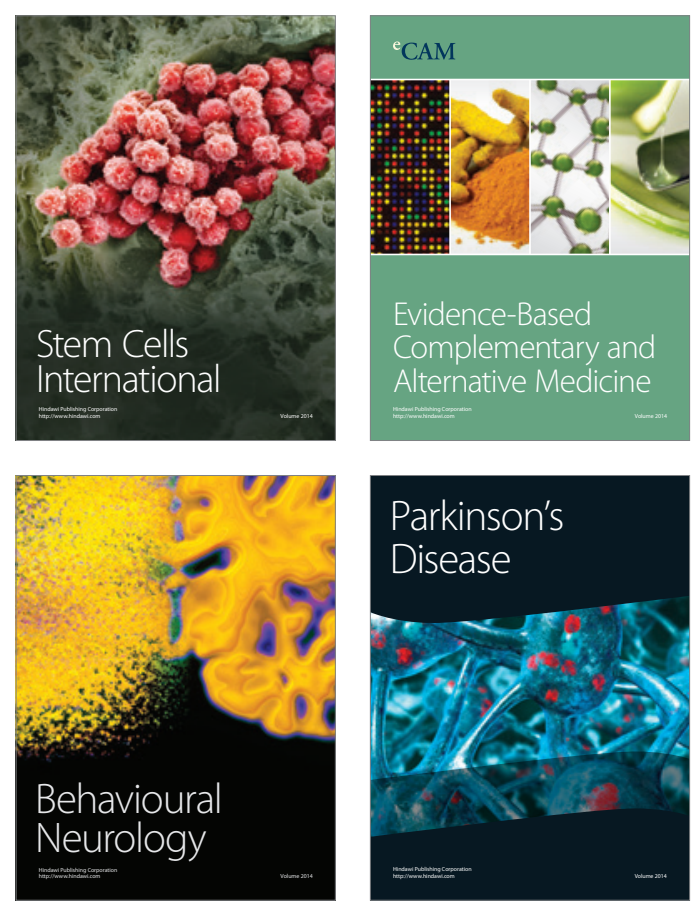

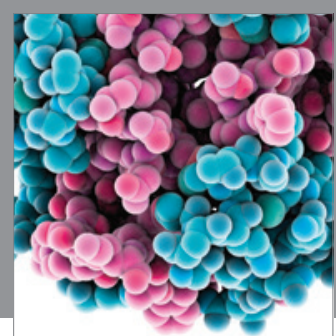

Journal of
Diabetes Research

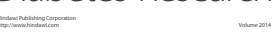

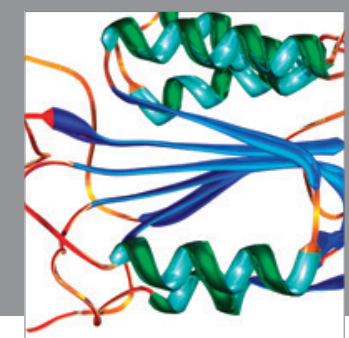

Disease Markers
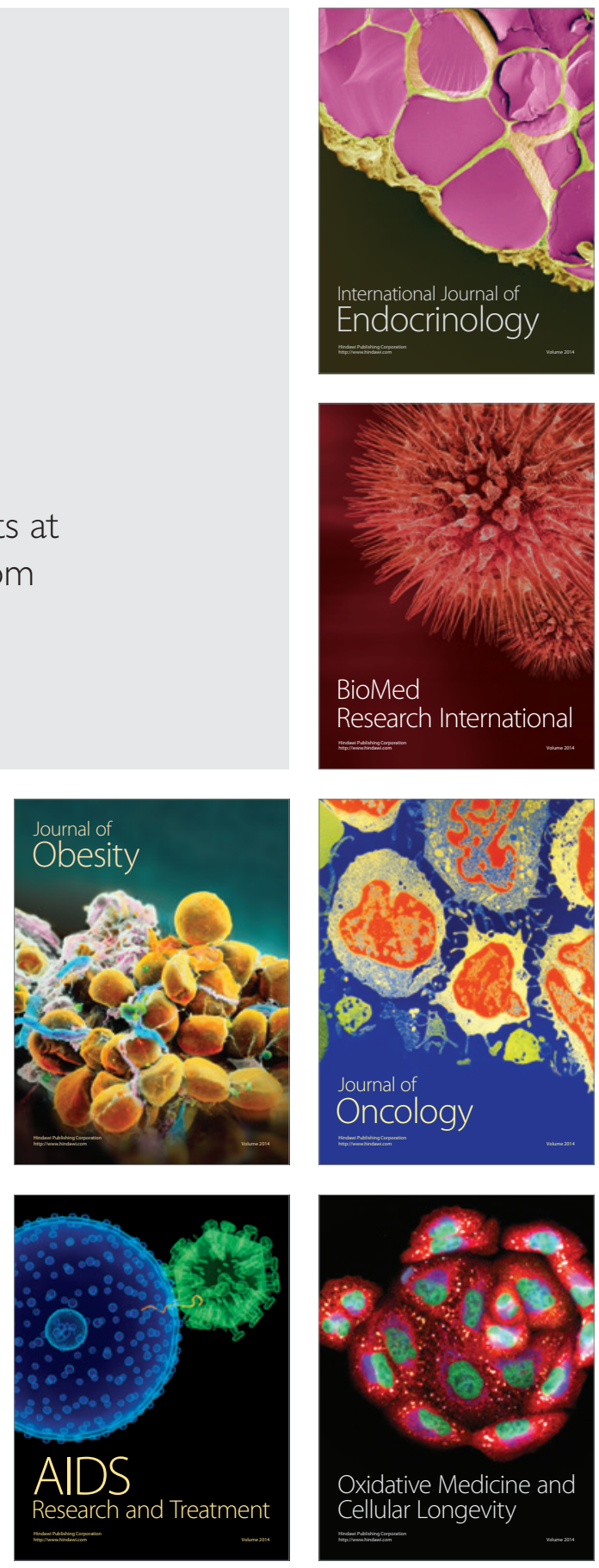